\title{
Recreational hobbies and their relationship with emotional intelligence for workers in sports recreation field at Mansoura University
}

\section{Hazem Mohammed Yousef Mansour}

Assistant professor at Sports Recreation Department, Physical Education Faculty, Mansoura University.

\section{Abstract}

The researcher carried out this research with the aim of identifying recreational hobbies and their relationship with emotional intelligence for workers in the field of sports recreation at Mansoura University, The researcher used the descriptive approach, and the Recreational Hobbies and Affective Intelligence scale was applied from the researcher's design to collect data, and the study was applied to a sample of( 225) students from Mansoura University for the basic experience and (50) for the exploratory experience. The most important results were the perception and awareness of most university employees of the concept of recreational hobbies The activity of browsing the Internet sites attracts the interest of most Employees, as it came at the forefront of the arrangement of recreational hobby activities, the presence of a medium level of recreational hobby activities and the emotional intelligence of the Employees, and the presence of a positive correlation between the practice of recreational hobbies and the emotional intelligence of the Employees.

\section{Introduction and Research Problem:}

The phenomenon of leisure and recreation has become part of the fabric of social systems that make up society, and interest in entertainment as a manifestation of civilized behavior began. Therefore, the interest in entertainment increased and its multiplicity to keep pace with the increasing increase in leisure time in contemporary societies and the increasing demand for entertainment to invest this time.

Muhammad Al Hamhami and Aida Abdel Aziz (2006) indicate that free time is when recreational hobbies, preferences and needs are satisfied, As well as developing talent, innovation and creativity, achieving psychological balance, and developing the human personality in general (10:18).

Recreation aims at the happiness that every individual seeks, regardless of gender, color, creed and happiness as a result of a life characterized by balance, and recreational hobbies have a distinctive place in making individuals' lives balanced between work and rest, so that life completes its meaning and becomes more joyful and brighter, . Recreational hobbies are a safe refuge for the health and time of individuals in modern societies, as they enable them to meet the needs and desires of the human soul, get rid of the pressures of working life, and highlight and discover the talents and abilities of different individuals. (19)

And emotional intelligence is one of the modern topics that have the interest of researchers. Affective intelligence is one of the most important types of intelligence that indicates the success of an individual in his personal life. Bilal Najma (2014) indicates that emotional intelligence is a group of diverse 
capabilities that individuals possess and are necessary for success in various aspects of social life that can be learned and improved, including emotional knowledge, managing emotions, enthusiasm, perseverance, motivation of the soul, awareness of others' emotions, awareness of social relationships. (5:14)

Safa al-Aasar and Ala al-Din Kafafi (2000) indicate that persons with high emotional intelligence are distinguished by a high communication skill, which means the ability to send and receive clear and convincing messages. Those who have this skill influence interaction with others and focus on the emotional connotations and their messages (14:54).

Alaa Abd al-Hadi (2013) points out that emotional intelligence has an important effect on the way people think about relationships and emotions, and there is a common denominator between sentiment and thinking and between the mind and the heart, and there is cooperation between them to allow people to make the right decisions and think properly about their emotions, control their emotions even They were of a high level of intelligence. (2: 102).

This is confirmed by Reem Sweilem (2018), that the weakness of emotional intelligence affects people in their lives and may lead to depression and living in an environment full of sadness, as well as the existence of a negative relationship between emotional intelligence and levels of depression, and from here it becomes clear how emotional intelligence affects people's behaviors and their relationships Socializing with others. (13:3).

Thus, the research problem crystallizes where the weakness of emotional intelligence generates a feeling of frustration in individuals and leads to a decrease in efficiency and self-efficacy, as well as limits the opportunities for creativity and thinking to solve problems systematically, in addition to a defect in social relations and affects psychological and social interaction with others.

Given the university's keenness and interest in developing students 'personality, investing their spare time in meaningful and useful programs, and working on employing recreational activities alongside the educational process so that students' personality can be developed in a balanced and comprehensive manner. In order for this to be achieved, there must be a human element capable of dealing with students in a manner and in a manner that contributes to attracting students and persuading them of the need to participate in recreational activities, This may only be achieved if these workers are distinguished by a high level of emotional intelligence in the way they deal with different students at the university level, In the case of a low level of emotional intelligence among workers in the field of sports recreation, this may be a major reason for students 'reluctance to participate in recreational activities as a result of the workers' inability to interact and communicate well with different groups of students and form successful social 
relationships.

Thus, the problem of research becomes clear and given that recreational hobbies provide a great deal of social, psychological and aesthetic skills, as well as a means of transferring experiences and developing thinking and intelligence, Therefore, the various recreational hobbies may have an important role in developing the emotional intelligence of the workers and encouraging them to think organized, work productively, innovation and creativity, and to show all the positives in the personality of persons, Where he can express everything inside as well as develop his mental capabilities and emotional intelligence, which leads to an individual feeling of self-confidence, a sense of comfort and cooperation with others and his ability to achieve success in his personal and practical life, which prompted the researcher to study recreational hobbies and their relationship with the emotional intelligence of workers in the field of sports recreation Mansoura University.

\section{Research purpose and questions:}

The research aims to identify the relationship between recreational hobbies and emotional intelligence for workers in the field of sports recreation at Mansoura University, by answering the following questions:

1. What is the level of recreational hobbies for workers in the field of sports recreation at Mansoura University?

2. What is the level of emotional intelligence for workers in the field of sports recreation at Mansoura University?

3. What is the correlation between recreational hobbies and emotional intelligence of workers in the field of sports recreation at Mansoura University?

4. What is the significance of the differences in recreational hobbies and emotional intelligence of workers in the field of sports recreation at Mansoura University, which are attributed to (gender, qualification, years of experience)?

\section{Search procedures:}

The researcher used the descriptive method in the survey method due to its relevance to the nature of the research, as well as the statistical approach that is consistent with the treatment of his study data that was collected through the Recreational Hobbies scale and the emotional intelligence scale.

\section{Research and Sample Society:}

The research community is represented by workers in the field of sports recreation at Mansoura University, The researcher selected the research sample randomly, and it consisted of (225) workers in the field of sports recreation at Mansoura University for the basic sample and (50) for the exploratory sample of workers in the field of sports recreation at Mansoura University and outside the basic sample . 


\section{Data collection strategy:}

1. Basic Data" form for the research sample. Appendix (1).

2. The scale of recreational hobbies scale consists of (80) singles distributed over (4) dimensions - prepared by the researcher Appendix (2).

3. The emotional intelligence scale consists of (62) items distributed on (4) dimensions - prepared by the researcher Appendix (3).

4. To identify the relationship between recreational hobbies and emotional intelligence of workers in the field of sports recreation at Mansoura University.

veracity test of the scale of recreational hobbies and emotional intelligence of workers in the field of sports recreation at Mansoura University.

To verify the suitability of the scale of recreational hobbies and emotional intelligence for workers in the field of sports recreation at Mansoura University, the researcher applied the two scales on an exploratory sample of (50) workers in the field of sports recreation at Mansoura University from within the community and outside the research sample in the period from (9/26/2020 AD) To 10/4/2020 $\mathrm{AD})$.

The researcher used the validity of internal consistency to calculate the validity of the dimensions of the scale of recreational hobbies and emotional intelligence of workers in the field of sports recreation at Mansoura University by finding the simple correlation coefficient of Person between the vocabulary belonging to each dimension by finding the correlation coefficient between each item and the total degree of the dimension as well as between the total degree of each dimension And the sum of the scale scores.

Stability of the scale of recreational hobbies and emotional intelligence of workers in the field of sports recreation at Mansoura University.

The reliability of the recreational hobby scale ranges between $(0.83-0.89)$, while the values of the affective intelligence scale ranged between $(0.79-0.89)$, which indicates the stability of the two scales.

Field study:

After reassurance of the honesty and consistency factors, the researcher applied the scale of recreation activities and the scale of motivation of learning to the basic study sample of (727) students from Mansoura University practicing recreational activities, in the period from $(17 / 2 / 2020 \mathrm{~m})$ to $(12 / 3 / 2020 \mathrm{AD})$, and after completing the application of the two measures, the data has been emptied in preparation for the appropriate statistical treatments.

Presenting and discussing the results:

What is the level of recreational hobbies for workers in the field of sports recreation at Mansoura University? 
From table (1), shows that the evaluative percentages of recreational hobbies for workers in the field of sports recreation at Mansoura University range from $(72.28 \%)$ to $(83.14 \%)$ with a high to medium degree of appreciation and weight. Of the scale as a whole it $(76.00 \%)$ is at an average level. This may be due to the awareness and realization of most workers that recreational hobbies are one of the purposeful and constructive activities that enable them to meet their needs and satisfy their various desires and motives, as well as knowing its importance in liberation and getting rid of life pressures and work burdens, in addition to the fact that recreational hobbies are characterized by fun and excitement and spreading an atmosphere of Fun and joy in the hearts of those who practice it . Appendix (4).

This is in agreement with the study of Mervat El-Gohary (2001) (9), Abdo Muhammad (2017), (1), ELzahaby M, Mansour N, Gouda H (2013) (7), Salwa AlHarbi (2010) (15), which clarified the perception and awareness of individuals of the concept of recreational activities and hobbies and the interest in practicing them in their spare time, because it is one of the activities that love their souls.

What is the level of emotional intelligence for workers in the field of sports recreation at Mansoura University?

From table (2), that the weighted percentage of the emotional intelligence scale for workers in the field of sports recreation at Mansoura University ranged between $(73.56 \%)$ to $(77.21 \%)$ with an average level of appreciation, The value of the percentage of the scale as a whole reached (75.52\%) at an average level, and this may be due to the keenness of most of the workers in the field of recreation at the university to have self-efficacy and enjoy personal skills, which are basic skills that help achieve success and professional and functional excellence such as establishing interactive relationships with others and bearing events And stressful situations, as well as having self-confidence and the ability to manage reality effectively . Appendix (5).

This is in agreement with the study of Sheng H, Tsour D (2017) (16), Waheeb Yasin (2016) (17), Rayburn, Pamela J (2013)(12), Faik Arduhan A (2012) (8), Mona Saeed (2002) (11), which showed the existence of a positive relationship between the sub-dimensions managing emotions, motivation, getting to know others, as well as individuals with high emotional intelligence are keen to enjoy personal competence, the ability to adapt, manage pressures, and establish close relationships with others.

\section{Correlation coefficient between recreational hobbies and the emotional intelligence of workers}

It is clear from table (3) that there is a statistically significant direct correlation between recreational hobbies and emotional intelligence of workers in the field of sports recreation at Mansoura University, as the calculated value of "R" 
is greater than its tabular value at a significant level (0.05), This may be due to the fact that recreational hobbies, in their various forms and multiple patterns, serve as an important and appropriate means for transferring experiences, developing thinking skills and intelligence among individuals and encouraging them to think positive and organized, as well as innovative and productive work, in addition to the fact that individuals' practice of recreational hobby activities helps in forming a human personality that has the ability to Determining his desires and personal motives and enabling him to feel good about himself, others and the society in which he lives, and thus be a socially balanced person who enjoys successful relationships with those around him and is characterized by a high degree of leadership, psychological and physical health. Appendix (6).

This is in agreement with the study of Bric Eirn(2020) (6), Ali Al-Qarni (2014) (3), Faik Ardohan (2012) (8), Aya Ayashi, Alan E (2006) ( 4), Mona Saeed (2002) (11), who showed the existence of a correlation between all dimensions of emotional intelligence, communication skills, flexibility in human relationships and social aspects, and that individuals who have a high level of intelligence prefer to engage in activities in their spare times.

What is the significance of the differences in recreational hobbies and emotional intelligence of workers in the field of sports recreation at Mansoura University, which are attributable to (gender)?

It is clear from table (4) that there are differences in the recreational hobbies of workers (males - females) in the field of sports recreation at Mansoura University in favor of males in the axis (motives for recreational hobbies recreational hobbies - the total score of the scale), and there are differences in the emotional intelligence of workers (males - Females) in the field of sports recreation at Mansoura University for the benefit of males in the axis of (personal motivation - self management - the total score of the scale),, Since the calculated value of " $\mathrm{T}$ " is greater than its tabular value at a significant level (0.05). This may be due to the interest of most male workers in the field of recreation and their desire to achieve their ambitions and aspirations at the professional and professional level and achieve success and excellence, in addition to the ability of male workers To deal with severe emotions with others and their ability to conform with others, to control negative emotions, and to effectively practice social and professional life. Appendix (7).

This is in agreement with the study of Bric Eirn (2020) (6), Ali Al-Qarni (2014) (3), Yoon J (2008) (18), Aya h, Alan E (2006) (4) ), Mona Saeed (2002) (11), who showed that there are statistically significant differences in terms of gender (males, females) and in favor of males.

What is the significance of the differences in recreational hobbies and 


\section{emotional intelligence of workers in the field of sports recreation at Mansoura University, which are attributable to (qualification)?}

Table (5) shows that there are differences in recreational hobbies and emotional intelligence of workers in the field of sports recreation at Mansoura University, which are attributed to (qualification) as the calculated value of " $q$ " is greater than its value. A tabular value at a significant level (0.05). Appendix (8).

The least significant difference in recreational hobbies and emotional intelligence of workers in the field of sports recreation Mansoura University, which is attributed to (qualification)

It is clear from table (6) that there are differences in the recreational hobbies of workers in the field of sports recreation at Mansoura University, which are attributed to (qualification), in favor of holders of a $\mathrm{PhD}$ in (the concept of recreational hobbies - recreational hobbies - the total score of the scale), this may be due to awareness and understand Most of the workers hold a doctorate degree with the importance of hobbies and recreational activities in satisfying their tendencies and desires, as well as information, knowledge and cognitive culture, which greatly helps them in exploiting free time in extracting their creative, intellectual and mental energies.

There are differences in the emotional intelligence of workers in the field of sports recreation at Mansoura University, which are attributed to (qualification), in favor of holders of a $\mathrm{PhD}$ qualification in (personal motivation - human relations management - self management - the total score of the scale), This may be due to the skills possessed by most workers with a higher qualification Ph.D. and have a sense of scientific research, knowledge and cultural weight that enriches the personality of workers to have a strong personality with effectiveness at the social level, in addition to the desire of workers with higher qualifications $\mathrm{PhD}$ to achieve self-esteem and enjoyment With an ambitious outlook for the future, and eagerness to achieve their aspirations and occupy a prestigious position befitting the educational level, and thus achieve success, professional and career excellence, and achieve social status) Appendix (9).

This is in agreement with the study of Yoon J (2008) (18), Aya Ayashi, and Allan Ayunt (2006) (4), which showed the existence of statistically significant differences for individuals with a good educational level.

What is the significance of the differences in recreational hobbies and emotional intelligence of workers in the field of sports recreation at Mansoura University, which are attributable to ( years of experience)?

It is clear from table (7) that there are differences in recreational hobbies and emotional intelligence of workers in the field of sports recreation at Mansoura University, attributed to (years of experience), as the calculated value of "q" is 
greater. Of its tabular value at a significant level (0.05). Appendix (10).

The least significant difference is in recreational hobbies and emotional intelligence for those working in the sports recreation field

table (8) shows that there are differences in the recreational hobbies of workers in the field of sports recreation at Mansoura University, which are attributed to (years of experience), in favor of those with the number of years of experience: 10 years or more in (motives for recreational hobbies - places of practice - the total score of the scale). This may be due to the amount of experience that workers have in the field of recreation at the university, as well as the abundance of knowledge and information related to recreational culture during the years of work, as well as the great familiarity with the places and regions that provide various services and various recreational activities), There are differences in the emotional intelligence of workers in the field of sports recreation at Mansoura University, which are attributed to (years of experience), in favor of those with the number of years of experience: 10 years or more "in (personal motivation - self management - emotional understanding - the total score of the scale), this may be due to The large number of situations and experiences that workers in the field of recreation have gone through for more than 10 years, their ability to deal with such situations and events in the appropriate manner, and their skills to solve problems in the appropriate manner as a result of their professional and practical experience. Appendix (11).

This is in agreement with the study of Yoon Hachung, Tasur Dishchenko (2017) (16), and Waheeb Yasin (2016) (17), Ali Al-Qarni (2014) (3), Aya Ayashi, Allan Ayunt, (2006) (4), which showed that there are statistically significant differences for individuals with years of work experience Mansoura University attributed to (years of experience)

\section{Research conclusion:}

Based on the results of this research and in light of the method used and within the limits of the sample tools and data collection, the researcher reached the following conclusions :

1. Realization and awareness of most workers in the field of recreation at the university of the concept of recreational hobbies, through the practice of its various activities.

2. The interest of most workers in the field of recreation at the university to achieve goals (cultural and physical) in order to develop physical and mental health, which achieves the restoration of psychological balance.

3. The activity of browsing the Internet sites attracts the interest of most workers, as it is at the forefront of arranging recreational hobby activities that employees accept to practice. 
4. There is a positive correlation between practicing recreational hobbies and emotional intelligence for workers in the field of recreation at Mansoura University.

5. The existence of statistically significant differences in the recreational hobbies of workers in the field of recreation at the university (males - females) in favor of males in the axis (motives for recreational hobbies - types of recreational hobbies) and the presence of differences in emotional intelligence in favor of males in the axis (personal motivation - self-management).

6. There are statistically significant differences in the emotional intelligence of workers in the field of sports recreation, which are attributed to (qualification), in favor of holders of a $\mathrm{PhD}$ in (personal motivation - management of human relations - self management), the existence of differences in recreational hobbies in favor of holders of a PhD in ( The concept of recreational hobbies recreational hobbies).

\section{Research recommendations:}

1. Realization and awareness of most workers in the field of recreation at the university of the concept of recreational hobbies, through the practice of its various activities.

2. The necessity to develop a plan to develop the cultural awareness of university employees in using free time in practicing recreational hobbies and to show the effect of this in providing a stable emotional environment during dealing with students at the university.

3. The interest of the university administration and officials in providing an appropriate university environment for workers in the field of recreation and providing opportunities for them to participate in hobbies and recreational activities in proportion to their needs and abilities.

4. It is necessary for the university to provide the recreational facilities and materials that workers need to practice their favorite recreational hobbies.

5. The necessity of activating in-service training programs to develop emotional intelligence skills and recreational hobbies, and that these programs be available to all workers in the field of recreation and leaders who are nominated to occupy leadership positions in the future.

\section{References}

1. Abdo Mohammed: Recreational interests in mobile phone technologies and their implications for spending leisure time among university youth, published research, Assiut Journal of Physical Education Science and Arts, Assiut University, Faculty of Physical Education, Issue 44, Part Three, 2017.

2. Alaa Abdel Hady: Metacognition skills and its relationship to self-efficacy and emotional intelligence among a sample of those with administrative jobs, Master Thesis, Faculty of Arts, Mansoura University, 2013 AD. 
3. Ali Al Qarani: Emotional Intelligence and its Relation to Communication Skills of Student Advisors, Master Thesis, College of Graduate Studies, Department of Educational Psychology, King Abdulaziz University, Saudi Arabia, 2014.

4. Aya h, Alan E: Outdoor Leaders Emotional Intelligence and Trans form ational Leadership, Journal of Experiential Education, Vol No 3, New York, 2006.

5. Bilal Najma: Emotional intelligence and its relationship to self-confidence among university students, a field study on a sample of university students, master's thesis, Faculty of Human and Social Sciences, University of Tizi Ouzou, Algeria, 2014.

6. Bric E: Developing EL: Leadership Joureys in Emotional Intelligence, M.S, Pepperdine University, Journal of ProQuest Dissertations, 2020.

7. ELzahaby M, Mansour N, Gouda H: Patterns of Recreational hobbies and their relationship to optimism, pessimism and socisl communication for the elderly, The 11th International Scientific conference for physical Education and Movement Sciences Sport in Theory, practice, faculty of physical education for boys, Alexandria University, 2013.

8. Faik A: Life Satisfaction and Emotional Intelligence of Participants, Non Participants in outdoor sports Turkey case, Journal of Procedia- Social and Behavioral Sciences, Vol No 62, New York, 2012.

9. Mervat El Gohary: The effect of practicing some extension activities and recreational hobbies on the development of social responsibility and its relationship to the attitudes of high school students towards the environment, published research, Journal of Comprehensive Education Research, College of Physical Education, Zagazig University, Volume One, 2001 AD.

10. Mohamed Al-Hamahm, Aida Abdulaziz: Recreation between theory and practice, 4th Edition, Cairo, AlKitab Center for Publishing, 2006 AD.

11. Mona Saeed: Emotional intelligence and its relationship to general intelligence, social skills and personality traits, a global study, published research, Cairo, The Egyptian Journal of Psychological Studies, Volume 12, Issue 25, 2002 AD.

12. Rayburn , Pamela J: Assessment of motivation and interest in outdoor recreation activities facilitated by a campus recreation center, Journal ProQuest Dissertations, 2013.

13. Reem Sweilem: Emotional Intelligence and its Relationship with Social Skills among Kindergarten Children, Master Thesis, Kuwait University, 2018.

14. Safaa Al-Aasar, Aladdin Kafafi: Emotional Intelligence, Quba House for Printing and Publishing, Cairo, 2000 AD.

15. Salwa Al-Harbi: Some types of recreation for third-grade secondary school students in Makkah Al-Mukarramah, field study, master's thesis, College of Education, Umm Al-Qura University, Saudi Arabia, 2010 AD.

16. Sheng H, Tsour D: The Effect of Tour Leaders, Emotional in telligence on Tourists, consequences, Journal of Travel Research, 2017.

17. Waheeb Yassin: Emotional Intelligence and its Relation to Professional Pressure Management for Trainers of Some Individual and Group Games, PhD Thesis, Faculty of Physical Education, Mansoura University, 2016.

18. Yoon J: Emotional Intelligence and Leadership Effectiveness: The Verbal and Nonverbal Communication Behaviors of Emotionally Intelligent Leaders, $\mathrm{PhD}$, City University of New York, New York, 2008.

19. www.new.edu.com 